\section{Antimicrobial Effect and Transdentinal Diffusion of New Intracanal Formulations Containing Nitrofurantoin or Doxycycline}

Ana Rita Silva ${ }^{1}$, Elizabete Brasil Santos², Shelon Cristina Souza Pinto², João Carlos Gomes ${ }^{2}$, Irene Pina Vaz ${ }^{1}$, Manuel Fontes Carvalho ${ }^{1}$
'Department of Endodontics, University of Oporto, Oporto, Portugal ${ }^{2}$ Department of Dentistry, UEPG - Ponta Grossa State University, Ponta Grossa, PR, Brazil

Correspondence: Ana Rita Marques da Silva, Rua Comandante Sacadura Cabral, $n^{\circ} 22$, 3030-333 Coimbra, Portugal. Tel: +351-91-248-1678. e-mail: anaritavlis@gmail.com

\begin{abstract}
The aim of this study was to investigate in vitro the antimicrobial effect and diffusion against $E$. faecalis of new intracanal medications on the external root surface. The medications tested were a placebo gel (PC); the new formulations with either 3\% nitrofurantoin (NIT) or 3\% doxycycline hydrochloride (DX) and $2 \%$ chlorhexidine (CHX) gel as positive control. The new formulations were tested using the traditional agar diffusion test (ADT) and an adapted agar diffusion method (AADM), where the teeth were filled with the medications and left to diffuse on agar surface seeded with E. faecalis. In the ADT, the larger zones of microbial growth inhibition were seen in DX, followed by CHX and NIT. In the AADM test only DX and CHX showed antimicrobial effect. Statistically significant differences between groups were observed by the Kruskal-Wallis test $(\chi 2=47.126 ; p<0.001)$. The new intracanal formulations with DX and NIT have demonstrated antimicrobial effect against $E$. faecalis, but only DX was able to diffuse through the dentinal tubules and exert antimicrobial effect outside the roots.
\end{abstract}

Key Words: Endodontics, enterococcus faecalis, doxycicline, nitrofurantoin.

\section{Introduction}

Endodontic infections are essentially polymicrobial and in most cases the microorganisms grow in the root canal system as sessile biofilms. A variety of bacterial species may be isolated from primary periapical infections. In the secondary infections, one of the most often isolated species is Enterococcus faecalis, which is seen as an opportunistic pathogen and a natural inhabitant of the oral cavity that survives as a mono-infection (1).

Despite the action of the chemomechanical preparation with antimicrobial irrigating solutions, some microorganisms, including $E$. faecalis, may survive in the root canal system. The use of an intracanal dressing that could act through the root canal system would certainly improve the outcome of the endodontic treatment (2).

The intracanal medication should have a broad antimicrobial spectrum, should be able to diffuse through the dentinal tubules and all ramifications of the root canal system including the areas that neither the instruments nor the irrigating solution can reach, and should not be cytotoxic to live tissues (3). Several substances have been tested and improved in order to achieve the ideal results. Calcium hydroxide $\left(\mathrm{Ca}(\mathrm{OH})_{2}\right)$ is believed to have most of the properties of an ideal root canal dressing due to its high $\mathrm{pH}$. However, it is not equally effective against all species of microorganism found in the root canal system (4).

Chlorhexidine gluconate has been used in endodontics as an irrigant. Recently, due to its antimicrobial effect and adsorption to dental hard tissues, it has also been suggested as an intracanal medication $(5,6)$. Authors have demonstrated that chlorhexidine is more effective than $\mathrm{Ca}(\mathrm{OH})_{2}$ when used against $E$. faecalis (5), and that the use of intracanal medication with chlorhexidine alone or associated with calcium hydroxide reduces the microbial factors responsible for pain and inflammation after endodontic treatment (7).

Antibiotics have been used in endodontics and for dental traumas systemically and locally. Although local application of antibiotics could cause side effects such as discoloration (8), this may be a more effective way for delivering the antibiotics (9) and reducing the bacterial load inside the root canal system, since the antibiotic agents increase significantly the antibacterial properties of endodontic materials (10). This effectiveness is due to the fact that it does not depend on circulation to reach the infected sites with compromised vasculature (11).

Recently, an alternative root canal irrigant - MTAD, a mixture of $3 \%$ doxycycline, $4,25 \%$ citric acid and $0,5 \%$ polysorbate 80 detergent (Tween 80 ) - was introduced as a final irrigant in the root canal preparation. Several studies concluded that it is a promising irrigating solution due to its biocompatibility (12) and clinical effectiveness against E. faecalis (13-15) and other microorganisms (16).

However, regarding the antimicrobial component of MTAD (doxycycline), some authors claim that nitrofurantoin, a synthetic nitrofuran commonly used in 
urinary tract infections where $E$. faecalis is usually involved $(17,18)$, has demonstrated better efficacy than doxycicline against the same bacteria (18).

The purpose of this experiment was to create an intracanal medication based on the formulation of MTAD. The better efficacy of nitrofurantoin to treat some $E$. faecalis infections when compared with doxycycline led the authors to choose it for the new formulation. The purpose of this study was to compare the antimicrobial effect of each new formulation against $E$. faecalis and its ability to diffuse through dentin.

\section{Material and Methods}

The antibacterial effect of the new formulations against E. faecalis was determined by an agar diffusion test (ADT) and an adapted agar diffusion method (AADM) through the external surface of the roots.

Agar Diffusion Test (ADT)

The methodology for the ADT was adapted from Vianna and Gomes (19). A 24-h culture of E. faecalis (ATCC 29203) in $\mathrm{BHI}$ agar was used to prepare a bacterial suspension containing $10^{8}$ viable bacterial cells $/ \mathrm{mL}$. The suspension was standardized at 0.5 in the spectrophotometer and McFarland scale. A sterile swab was used to place $E$. faecalis suspension on Muller Hinton agar plates. After drying for $10 \mathrm{~min}$ at $36^{\circ} \mathrm{C}$, a sterile glass tube was used to create wells ( $4 \mathrm{~mm}$ diameter $\times 4 \mathrm{~mm}$ depth) in the agar. The wells were filled with the substances described below:

- Placebo gel (PC) (50\% propylene glycol; 0.5\% polysorbate $80 ; 0.76 \%$ anhydrous monobasic sodium phosphate $\left(\mathrm{NaH}_{2} \mathrm{PO}_{4}\right) ; 1.94 \%$ anhydrous disodium phosphate $\left(\mathrm{Na}_{2} \mathrm{HPO}_{4}\right)$, and $41.8 \mathrm{~g}$ of $2 \%$ hydroxyethylcellulose gel)

- 2\% clorhexidine (CHX) $10 \mathrm{~g}$ of 20\% clorhexidine digluconate; $50 \%$ propylene glycol; $0.5 \%$ polysorbate 80 , and $39.5 \mathrm{~g}$ of $2 \%$ hydroxyethylcellulose gel)

- 3\% nitrofurantoin (NIT) (3g of nitrofurantoin; 50\% propylene glycol; $0.5 \%$ polysorbate $80 ; 0.76 \% \mathrm{NaH}_{2} \mathrm{PO}_{4}$; $1.94 \% \mathrm{Na}_{2} \mathrm{HPO}_{4}$, and $41.8 \mathrm{~g}$ of $2 \%$ hydroxyethylcellulose gel)

- 3\% doxycycline (DX) $3.33 \mathrm{~g}$ of doxycycline hydrochloride; $0.1 \mathrm{~g}$ of sodium metabisulfite; $50 \%$ propylene glycol; $0.5 \%$ polysorbate $80 ; 0.76 \% \quad \mathrm{NaH}_{2} \mathrm{PO}_{4} ; 1.94 \%$ $\mathrm{Na}_{2} \mathrm{HPO}_{4}$, and $43.37 \mathrm{~g}$ of $2 \%$ hydroxyethylcellulose gel)

After incubation at $36{ }^{\circ} \mathrm{C}$ for $24 \mathrm{~h}$, the zones of microbial growth inhibition were measured and recorded. The inhibitory zone was considered the smaller distance between the outer margin of the well and the beginning of the microbial growth. Means and standard deviations were used to compute the differences in efficacy of the tested substances.

\section{Adapted Agar Diffusion Method (AADM)}

The methodology used for AADM was a modification 426 of the one previously described by Gomes et al. 2003 and 2009. Fifty extracted human canine teeth were selected and the single root canal was confirmed radiographically. Curettes were used to remove soft tissues and debris from tooth surfaces and if any kind of anomaly, such as a fracture were detected, the tooth was excluded from the study. Teeth crowns were removed $3 \mathrm{~mm}$ above the cementoenamel junction and the roots were standardized to a length of $15 \mathrm{~mm}$ with a water-cooled diamond saw. A size $10 \mathrm{~K}$-file (Dentsply/Maillefer, Ballaigues, Switzerland) was introduced into each canal until it could be seen at the apical foramen. The working length was established subtracting $1 \mathrm{~mm}$ from this measurement $(14 \mathrm{~mm})$. In order to preserve patency, the same K-file was used to recapitulate the canal $1 \mathrm{~mm}$ beyond its length between each file. The anatomic diameter of apical foramen was sized using manual K-files, beginning with size 10 files, inserted until the working length was reached. File sizes were progressively increased until an instrument that bound at the working length was obtained. The size of the obtained instrument was recorded for each root. The roots were enlarged with $\mathrm{K}$-files three numbers up to apical file size determined at the working length

The teeth were instrumented using the crown-down principles, combining Gates Glidden drills (Dentsply/ Maillefer) with K-files. The cervical two-thirds $(10 \mathrm{~mm})$ of the canals were prepared using K-files 25 to 35 , followed by a size 2 Gates Glidden bur and then by a size 3,1 mm shorter. The apical stop was established using files three numbers up to the anatomic diameter file. At each file change the root canals were irrigated with $1 \mathrm{~mL}$ of 5,25\% $\mathrm{NaOCl}$. After mechanical preparation, the teeth were rinsed with $5 \mathrm{~mL}$ of $17 \%$ ethylenediamine tetra-acetic acid (EDTA) solution for $3 \mathrm{~min}$, to remove the smear layer, followed by $1 \mathrm{~mL}$ of $5,25 \% \mathrm{NaOCl}$ and finally with $5 \mathrm{~mL}$ of saline.

The teeth were sterilized in individual tubes containing $5 \mathrm{~mL}$ of Brain Heart Infusion Broth (BHI) and the process confirmed by overnight incubation of the specimens at 36 ${ }^{\circ} \mathrm{C}$. Then, they were divided in 5 groups of 10 teeth each, according to the substance used as intracanal dressing (20), which were the same described in the agar diffusion test (ADT): a) Teeth sealed without medication (WM - negative control); b) CHX - positive control, c) PC; d) NIT and e) DX.

Immediately after filling the root canals, the foramen and the pulp chamber were sealed with wax. The Muller Hinton agar plates were first seeded by a swab with the E. faecalis $10^{8}$ cells $/ \mathrm{mL}$ suspension and the teeth were then placed inside. The plates were kept for $2 \mathrm{~h}$ at room temperature to allow the diffusion of the agents through the dentinal tubules and the agar, and then incubated at 36 ${ }^{\circ} \mathrm{C}$ for $48 \mathrm{~h}$. After this period the zones of microbial growth inhibition were measured, considering the shortest distance 
from the outer margin of the tooth to the initial growth.

The non-parametric Kruskal-Wallis test was used to verify any significant difference between groups. In the post-hoc procedures the Tamhane test was used for multiple group comparisons, allowing the assumption that variances are not equal and adjustment for type I errors.

\section{Results}

\section{Agar Diffusion Test (ADT)}

Figure 1 and Table 1 show that, with the exception of the PC, all tested formulations had potential to eliminate E. faecalis. DX was the one that exhibited the largest inhibition zone followed by NIT and CHX.

\section{Adapted Agar Diffusion Method (AADM)}

Figure 2 shows the area of microbial inhibition of the different intracanal medications through the external

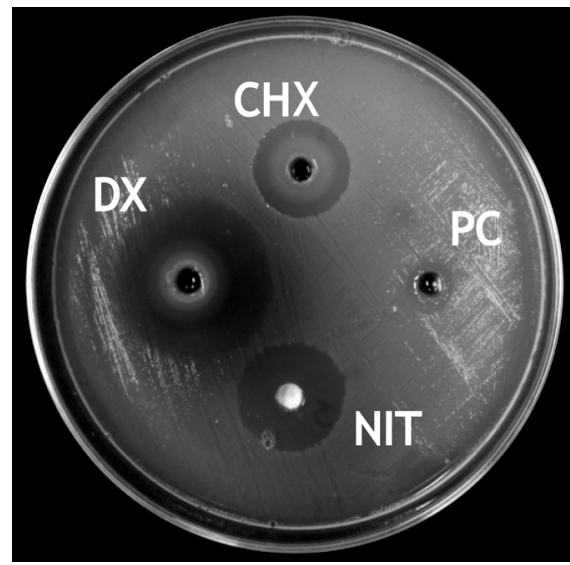

Figure 1. Zones of microbial growth inhibition $(\mathrm{mm})$ in ADT of the intracanal dressings against E. faecalis (CHX: $2 \%$ chlorhexidine; PC: Placebo; NIT: 3\% nitrofurantoin and DX: 3\% doxycycline). root surface against $E$. faecalis. Only CHX and DX showed inhibition zones, measuring $11.17( \pm 2.17) \mathrm{mm}$ and $5.52( \pm 2.9) \mathrm{mm}$ respectively, while PC and NIT did not inhibit E. faecalis growth as well as the negative control (WM). The Kruskal-Wallis test $(\chi 2=47.126 ; p<0.001)$ revealed statistical differences between the groups (Fig. 3). In a multiple comparisons post-hoc procedure it was possible to observe statistically significant differences between $\mathrm{CHX}$ and DX and the other groups.

\section{Discussion}

In both tests (ADT and AADM) DX presented the largest zones of microbial growth inhibition, demonstrating a better antibacterial effect and/or a better diffusion ability compared with NIT and CHX. NIT was not able to exert any antimicrobial action on the external root surface (AADM) during the incubation period. With the methodology used in this study, when the mean area of microbial inhibition is equal to zero, it means either the medication did not present any antibacterial effect or the substances were not able to diffuse through the agar or through the dentinal tubules. When ADT was applied, DX, NIT and CHX were effective in inhibiting $E$. faecalis growth, as also shown

Table 1. Mean and standard deviation of inhibition zone $(\mathrm{mm})$ of $E$. faecalis growth produced by the intracanal medication used in the experimental groups

\begin{tabular}{lc}
\hline Intracanal medication & Inhibition zones $(\mathrm{mm})$ \\
\hline $2 \%$ chlorhexidine & $20.70( \pm 0.30)$ \\
Placebo gel & $0( \pm 0.0)$ \\
$3 \%$ nitrofurantoin & $24.33( \pm 0.10)$ \\
$3 \%$ doxycycline hydrochloride & $36.27( \pm 0.24)$ \\
\hline
\end{tabular}
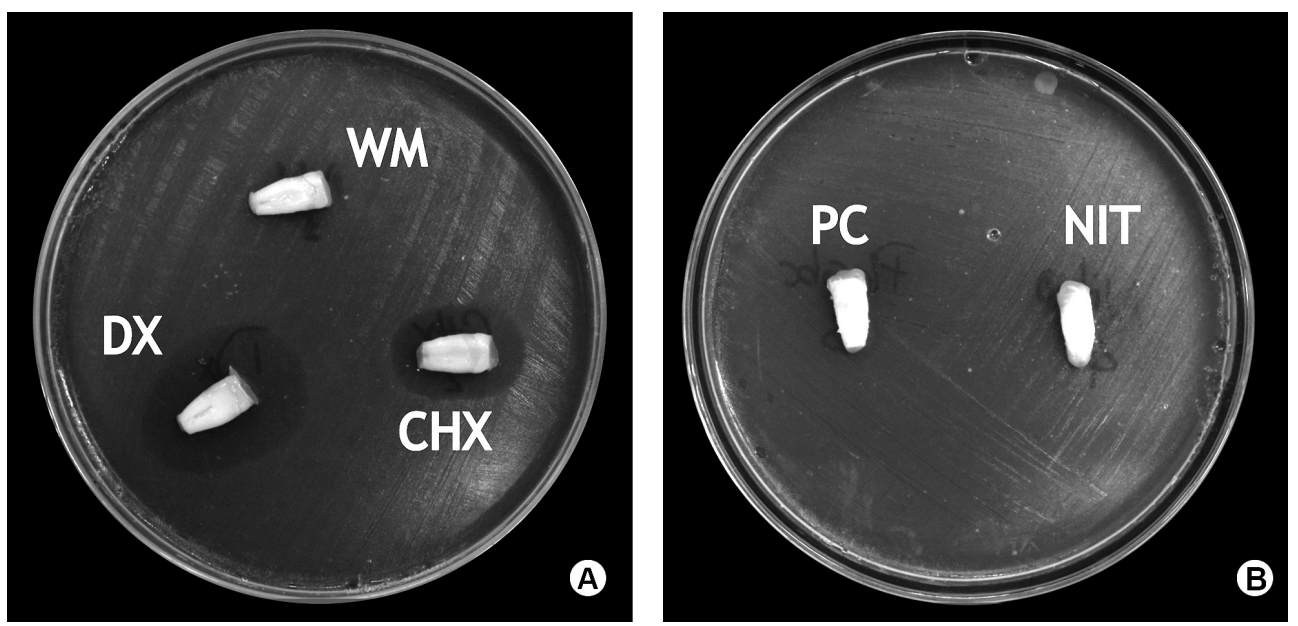

Figure 2. Zones of microbial growth inhibition $(\mathrm{mm})$ formed by the studied intracanal medications against $E$. faecalis after diffusion through dentinal tubules. a) WM - without medication; CLX: 2\% chlorhexidine and DX: 3\% doxycycline; b) PC: placebo and NIT: 3\% nitrofurantoin. 
by other authors (17).

Although the size of the inhibition zones would indicate activity against the microorganisms, their effectiveness depends on many factors, like the molecular size, solubility and diffusion of the materials through the agar medium, sensitivity of the drug, bacterial source, the number of inoculated bacteria, $\mathrm{pH}$ of the substrate in plates, agar viscosity, storage conditions of the agar plates, time of incubation and the metabolic activity against the microorganisms $(4,21)$.

In the AADM test the cementum layer could affect the diffusion of substances to the agar. However, some authors observed that presence or absence of cementum layer does not interfere significantly in the inhibition zones (20).

The NIT formulation presented a higher viscosity than DX. Although it demonstrated antimicrobial effect against E. faecalis in the ADT, this factor could have prevented it from reaching the external root surface, explaining the lack of diffusion observed in AADM. Therefore, it was understood that the methods applied in the present study could be used to analyze and verify the existence of antibacterial activity and diffusion ability trough dentinal tubules, but further studies are required.

The detergent used in intracanal formulations, Tween 80, reduces the surface tension allowing an increased wetting effect, and consequently a better penetration of

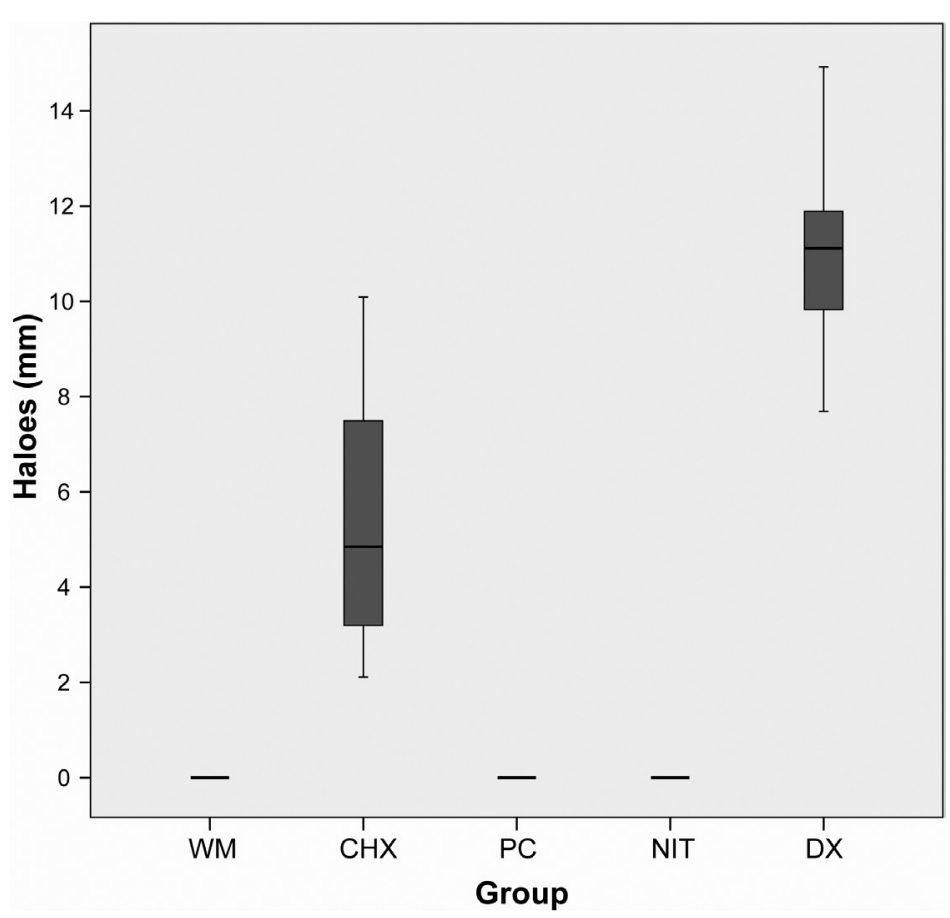

Figure 3. Box-plot graphic of the zones of microbial growth inhibition $(\mathrm{mm})$ formed by the different intracanal medications after $24 \mathrm{~h}$ of diffusion through canal dental system. Kruskal-Wallis test $(\chi 2=47.126 ; p<0.001)$. WM: without medication; CLX: 2\% chlorhexidine; PC: Placebo; NIT: 3\% nitrofurantoin and DX: 3\% doxycycline. intracanal medication into dentinal tubules and irregular spaces with difficult access (22). Since NIT presented still a high viscosity, the authors believe that it may be beneficial to increase the detergent weight percentage in the medication to observe better antimicrobial results outside the roots.

Since the low $\mathrm{pH}$ of MTAD induces dentin demineralization (23), in this study a phosphate buffer was used to induce a neutral $\mathrm{pH}$, with the goal of keeping the new formulations inside the root canals for longer periods.

The main purpose of this study was to create an intracanal medication based on MTAD with good activity against $E$. faecalis $(13,14)$, respecting the requirements of an ideal medication.

Chlorhexidine $2 \%$ is used as the gold standard for inhouse assay due to its broad spectrum and effectiveness against $E$. faecalis (24). For the $\mathrm{CHX}$ group, concentration of $2 \%$ was chosen because its effectiveness is well established. In the new formulations containing DX or NIT, concentration of $3 \%$ was used since they were based on MTAD solution.

The use of antibiotics in irrigant solutions made it possible creating new formulations of intracanal dressings using these products.

In the present study, the formulation with DX was the most effective against $E$. faecalis, showing larger inhibition zones than NIT. Nitrofurantoin is effective against many Gram positive and negative bacteria, including $E$. faecalis $(17,18)$ and doxycycline is a tetracycline broad-spectrum antibiotic, effective against a wide range of microorganisms (22). In some studies, DX showed low activity against most strains of $E$. faecalis (25). These results could also be explained by the use of a strain more susceptible to doxycycline than nitrofurantoin and this characteristic deserves further investigation.

Despite the limitations of this study, such as the fact that in vitro models present different conditions from in vivo infections and the fact that a mono-infection assay ( $E$. faecalis) was used to test the intracanal medication, the results are promising and indicate the efficacy of a new intracanal formulation proposed by the authors. Further studies are required to corroborate the present findings and expand the efficacy of these formulations.

The findings of this study suggest that both the new formulations with DX or NIT presented antimicrobial effect against $E$. faecalis. However, only DX was able to reach the dentinal tubules. These new formulations may be promising as intracanal dressings, increasing the outcome of 
the endodontic treatment in persistent infections

\section{Resumo}

0 objetivo deste estudo foi investigar, in vitro, o efeito antibacteriano e a difusão frente ao $E$. faecalis, de novas medicações intracanal na superfície externa da raiz. As medicações testadas foram um gel placebo (PC), as novas formulações quer com nitrofurantoina a $3 \%$ (NIT) ou hidrocloridrato de doxiciclina a 3\%(DX) e um gel de clorexidina a $2 \%$ (CHX) como controle positive. As novas formulações foram testadas usando o tradicional teste de difusão em agar (ADT) e um método de difusão adaptado (AADM), onde os dentes foram preenchidos com as medicações e deixados a difundir numa superficie de agar semeada com E. faecalis. No ADT, a maior área de inibição foi registada para DX, seguida por CHX e NIT. No teste AADM, apenas DX e CHX demonstraram ação antimicrobiana. Foram observadas diferenças significativas entre os grupos através do teste Kruskal-Wallis $(\chi 2=47.126 ; p<0.001)$. As novas formulações intracanais contendo DX e NIT demonstraram ação antimicrobiana quando em contacto com $E$. faecalis, mas apenas DX teve capacidade de difundir através dos túbulos dentinários e exercer ação antimicrobiana fora das raizes.

\section{Acknowledgements}

The authors would like to thank Prof. Dr. Fábio Andre Santos for his help with the statistical analysis and Prof. Dr. Paulo Vitor Farago for technical support.

\section{References}

1. Siqueira JF Jr., Rocas IN. Polymerase chain reaction-based analysis of microorganisms associated with failed endodontic treatment. Oral Surg Oral Med Oral Pathol Oral Radiol Endod 2004;97:85-94.

2. Athanassiadis B, Abbott PV, Walsh $\amalg$. The use of calcium hydroxide, antibiotics and biocides as antimicrobial medicaments in endodontics. Aust Dent J 2007;52:64-82.

3. Alencar $A H$, Leonardo MR, Silva LA, Silva RS, Ito IY. Determination of the $\mathrm{p}$-monochlorophenol residue in the calcium hydroxide + P-monochlorophenol combination used as an intracanal dressing in pulpless teeth of dogs with induced chronic periapical lesion. J Endod 1997;23:522-524.

4. Gomes BP, Ferraz CC, Garrido FD, Rosalen PL, Zaia AA, Teixeira FB, et al.. Microbial susceptibility to calcium hydroxide pastes and their vehicles. J Endod 2002;28:758-761.

5. Gomes BP, Souza SF, Ferraz CC, Teixeira FB, Zaia AA, Valdrighi L, et al.. Effectiveness of $2 \%$ chlorhexidine gel and calcium hydroxide against Enterococcus faecalis in bovine root dentine in vitro. Int Endod J 2003;36:267-275.

6. Delgado RJ, Gasparoto $T H$, Sipert CR, Pinheiro CR, de Moraes IG, Garcia RB, et al.. Antimicrobial activity of calcium hydroxide and chlorhexidine on intratubular Candida albicans. International Journal of Oral Science 2013;5:32-36.

7. Singh RD, Khatter $\mathrm{R}, \mathrm{Bal} R \mathrm{RK}, \mathrm{Bal} \mathrm{CS}$. Intracanal medications versus placebo in reducing postoperative endodontic pain--a double-blind randomized clinical trial. Braz Dent J 2013;24:25-29.

8. Day PF, Duggal MS, High AS, Robertson A, Gregg TA, Ashley PF, et al.. Discoloration of teeth after avulsion and replantation: results from a multicenter randomized controlled trial. J Endod 2011;37:1052-1057.

9. Mohammadi Z. Antibiotics as intracanal medicaments: a review. J Calif
Dent Assoc 2009:37:98-108.

10. Sharma D, Grover R, Pinnameneni PS, Dey S, Raju PR. Evaluation of efficacy of combinations of five endodontic sealers with five antibiotics against Enterococcus faecalis - An in-vitro study. Journal of International Oral Health: JIOH 2014;6:90-95.

11. Gilad JZ, Teles R, Goodson M, White RR, Stashenko P. Development of a clindamycin-impregnated fiber as an intracanal medication in endodontic therapy. J Endod 1999;25:722-727.

12. Singla MG, Garg A, Gupta S. MTAD in endodontics: an update review. Oral Surg Oral Med Oral Pathol Oral Radiol Endod 2011;112:70-76.

13. Torabinejad M, Shabahang $S$, Aprecio RM, Kettering JD. The antimicrobial effect of MTAD: an in vitro investigation. J Endod 2003;29:400-403.

14. Davis JM, Maki J, Bahcall JK. An in vitro comparison of the antimicrobial effects of various endodontic medicaments on Enterococcus faecalis. J Endod 2007;33:567-569.

15. Rizvi A, Zafar MS, Farid WM, Gazal G. Assessment of antimicrobial efficacy of MTAD, sodium hypochlorite, EDTA and chlorhexidine for endodontic applications: An in vitro study. Middle-East Journal of Scientific Research 2014;21:353-357.

16. Tong Z, Zhou L, Kuang R, Lv H, Qu T, Ni L. In vitro evaluation of MTAD and nisin in combination against common pathogens associated with root canal infection. J Endod 2012;38:490-494.

17. Al-Zarouni M, Senok A, Al-Zarooni N, Al-Nassay F, Panigrahi D. Extended-spectrum beta-lactamase-producing Enterobacteriaceae: in vitro susceptibility to fosfomycin, nitrofurantoin and tigecycline. Med Princ Pract 2012;21:543-547.

18. Butt T, Leghari MJ, Mahmood A. In-vitro activity of nitrofurantoin in enterococcus urinary tract infection. J Pak Med Assoc 2004;54:466469.

19. Vianna MEandGomes BP. Efficacy of sodium hypochlorite combined with chlorhexidine against Enterococcus faecalis in vitro. Oral Surg Oral Med Oral Pathol Oral Radiol Endod 2009;107:585-589.

20. Gomes BP, Montagner F, Berber VB, Zaia AA, Ferraz CC, Almeida JF, et al.. Antimicrobial action of intracanal medicaments on the external root surface. J Dent 2009;37:76-81.

21. Salem-Milani A, Balaei-Gajan E, Rahimi S, Moosavi Z, Abdollahi A, Bolourian $M$, et al.. Antibacterial effect of diclofenac sodium on Enterococcus faecalis. Journal of Dentistry, Tehran University of Medical Sciences 2013;10:16-22.

22. Torabinejad M, Johnson WB, inventors; Dentsply International (York, $\mathrm{PA})$, assignee. Irrigation solution and methods for use. United States patent 20030235804. 2003 December 25.

23. Wikesjo UM, Baker PJ, Christersson LA, Genco RJ, Lyall RM, Hic S, et al.. A biochemical approach to periodontal regeneration: tetracycline treatment conditions dentin surfaces. J Periodontal Res 1986;21:322329.

24. Sinha N, Patil S, Dodwad PK, Patil AC, Singh B. Evaluation of antimicrobial efficacy of calcium hydroxide paste, chlorhexidine gel, and a combination of both as intracanal medicament: An in vivo comparative study. J Conserv Dent 2013;16:65-70.

25. Cunha BA. New uses for older antibiotics: nitrofurantoin, amikacin, colistin, polymyxin B, doxycycline, and minocycline revisited. Med Clin North Am 2006;90:1089-1107. 